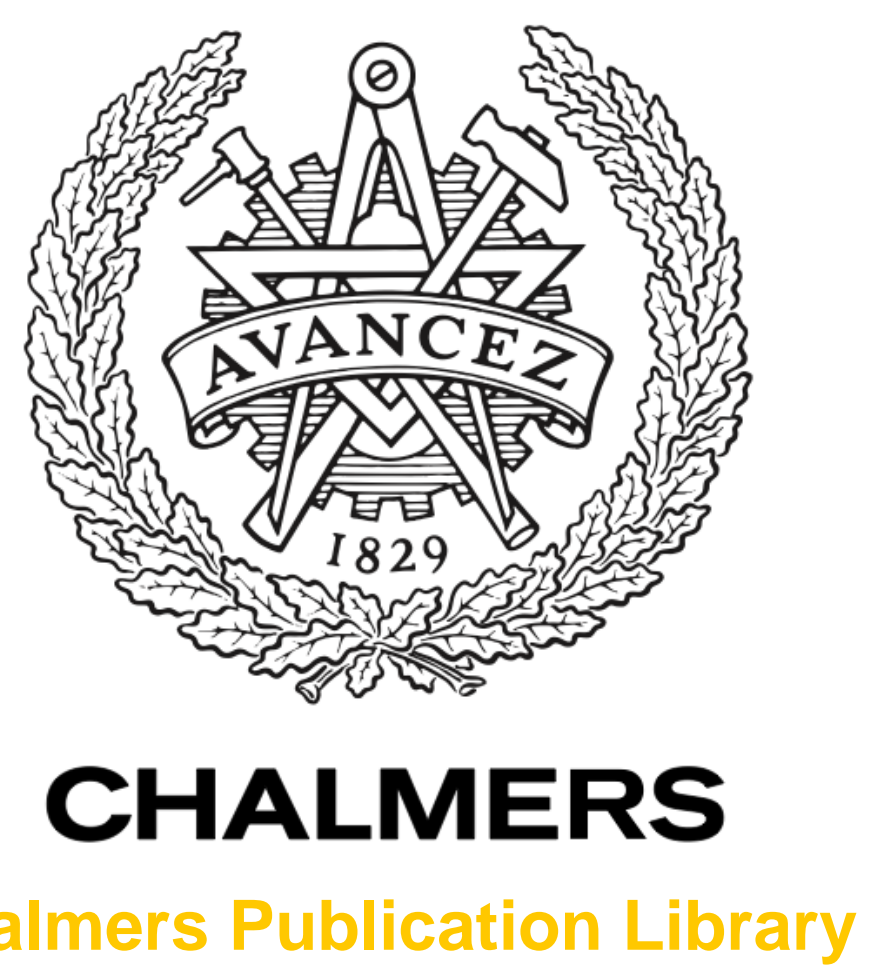

Challmers Publication Library

\title{
A hierarchy of dynamic equations for solid isotropic circular cylinders
}

This document has been downloaded from Chalmers Publication Library (CPL). It is the author's version of a work that was accepted for publication in:

Wave motion (ISSN: 0165-2125)

Citation for the published paper:

Abadikhah, H. ; Folkow, P. (2014) "A hierarchy of dynamic equations for solid isotropic circular cylinders". Wave motion, vol. 51(2), pp. 206-221.

http://dx.doi.org/10.1016/j.wavemoti.2013.06.010

Downloaded from: http://publications.lib.chalmers.se/publication/193563

Notice: Changes introduced as a result of publishing processes such as copy-editing and formatting may not be reflected in this document. For a definitive version of this work, please refer to the published source. Please note that access to the published version might require a subscription. 


\title{
A hierarchy of dynamic equations for solid isotropic circular cylinders
}

\author{
Hossein Abadikhah, Peter D. Folkow*, \\ Department of Applied Mechanics, Chalmers University of Technology, SE-412 96 \\ Göteborg, Sweden
}

\begin{abstract}
This work considers homogeneous isotropic circular cylinders adopting a power series expansion method in the radial coordinate. Equations of motion together with consistent sets of end boundary conditions are derived in a systematic fashion up to arbitrary order using a generalized Hamilton's principle. Time domain partial differential equations are obtained for longitudinal, torsional, and flexural modes, where these equations are asymptotically correct to all studied orders. Numerical examples are presented for different sorts of problems, using exact theory, the present series expansion theories of different order, and various classical theories. These results cover dispersion curves, eigenfrequencies and the corresponding displacement and stress distributions, as well as fix frequency motion due to prescribed end displacement or lateral distributed forces. The results illustrate that the present approach may render benchmark solutions provided higher order truncations are used, and act as engineering cylinder equations using low order truncation.
\end{abstract}

Key words: beam, torsion, series expansion, asymptotic, eigenfrequency

\section{Introduction}

Dynamic equations of circular cylinders have been studied extensively by many authors; from the full three dimensional geometry to simple one-dimensional models. Exact solutions to the three dimensional equations were derived by Pochhammer [1] and Chree [2] for

\footnotetext{
* Corresponding author. Tel: +46 31 7721521, Fax: +46 317723827.

Email addresses: hossein.abadikhah@chalmers.se (Hossein Abadikhah), peter.folkow@chalmers.se (Peter D. Folkow).
} 
infinitely long cylinders, resulting in the well-known transcendental frequency equations. Further investigations of these frequency equations have been carried out for the various mode families (longitudinal, torsional, flexural). Studies of the corresponding dispersion curves have been investigated in detail $[3,4,5]$ where results have been presented for the modes that are of most importance to the present work: the transverse lowest flexural modes and the higher order flexural mode families. Considering semi-infinite and finite cylinders, analytical transient solutions have been developed for the flexural modes in the special case of mixed boundary conditions $[6,7]$. Due to the complexity of the three dimensional theory of elastodynamics in general, and dealing with other end boundary conditions in particular (e.g. Dirichlet or Neumann), various approximate methods of solutions have been developed. There exists on one hand analytical solutions based on expansion in terms of Bessel functions $[8,9]$ where part of the boundary conditions are satisfied approximately, and on the other hand numerical solutions such as the Ritz method $[10,11,12,13]$ or the finite element method [14]. These works using approximate methods concentrate mainly on eigenfrequency analyzes.

However, the bulk of analysis on flexural problems has been directed towards the lowest transverse flexural mode family using simplified one-dimensional beam theories. In these simplified theories, both the dynamic equations and the boundary conditions are often derived using various kinds of simplifying kinematic assumptions. The most used approximate theory is the simple Euler-Bernoulli equation, where shear and rotary inertia are neglected. This leads to a differential equation that has the undesired feature of being non-hyperbolic. However, if the beam radius is much smaller than both the beam length and the wavelengths, this approximation is known to yield accurate results. The next level of models include shear and rotary inertia described by Timoshenko [15], resulting in a hyperbolic equation of motion. There are several other more advanced beam theories in use. Some of these concern only rectangular cross sections [16, 17, 18, 19, 20, 21, 22, 23], while others are applicable for circular cross sections [24, 25, 26, 27, 28, 29].

The present paper aims at systematically develop a hierarchy of approximate equations for solid isotropic circular cylinders. To this end power series expansions in the radial coordinate are adopted in the three dimensional equations of motion. Using generalized Hamilton's principle, time domain equations of motions together with general lateral and end boundary conditions are stated in a systematic manner. Sets of cylinder equations may hereby be derived to an (in principle) arbitrary order for the various displacement families (longitudinal, torsional, flexural), where each studied truncated order is asymptotically correct. Higher order sets of time domain equations may be used for benchmark solutions to various three dimensional cylinder problems, while lower order sets may be used as alternative engineering equations. As the longitudinal axisymmetric motion is investigated in a separate paper by Folkow and Mauritsson [30], the main contribution here concerns torsional and the more involved flexural motions. Especially the lowest flexural transverse modes are studied in some detail. 
Other higher order power series expansion flexural theories are presented in the literature $[24,25,26,28,20]$. Here, Martin [28] uses an approach on cylindrically anisotropic cylinders that in many respects is similar to the present method. The other cited works use approaches that are different from the present one concerning the series expansion method, the use of recursion relations, the procedure when collecting terms or the truncation process as a whole. Consequently, the resulting flexural theories (equations of motion, boundary conditions) are all different, even for the lowest truncation order. Besides solid cylinders, the present method has been adopted on shells and plates [31, 32, 33, 34, 35, 36].

Sections 2-6 illustrate the fundamentals of the method in question. Adopting the three dimensional equations of motion and a series expansion assumption, a hierarchy of variationally consistent approximate equations of motion and pertinent boundary conditions are derived from Hamilton's principle. The issue whether these sets of equations are asymptotically correct or not are illustrated and discussed in Section 7. The rest of the paper concentrates mainly on the flexural motion. Section 8 presents and discusses the differential equation for the present transverse beam theory using the lowest truncation order, together with equations for other beam theories given in the literature. Numerical results are given in Section 9, covering dispersion curves, eigenfrequencies, mode shapes, motion due to prescribed end displacement and a static deflection case. For the present theory, these examples illustrate both the benchmark property of the higher order truncations, and the efficiency of the lower order engineering equations.

\section{Hamilton's principle}

Consider a circular cylinder with length $L$ and radius $a$. The cylinder is homogeneous, isotropic and linearly elastic with density $\rho$ and Lamé parameters $\lambda$ and $\mu$. Cylindrical coordinates are used with radial coordinate $r$, circumferential coordinate $\theta$ and axial coordinate $z$. The corresponding radial, circumferential and longitudinal displacement fields are denoted by $u, v$ and $w$.

A generalized Hamilton's principle is to be used to derive variationally consistent sets of differential equations describing the motion of the cylinder together with the corresponding boundary conditions. Hereby, the governing sets of cylinder equations are treated in a unified manner. Simultaneous and independent variations of displacements and stresses are adopted such that displacement and stress boundary conditions are treated similarly $[30,37,38]$. The Hamilton's principle thus results in the variational expressions

$$
\int_{t_{0}}^{t_{1}}\left(\int_{V}(\nabla \cdot \boldsymbol{\sigma}+\rho \boldsymbol{f}-\rho \ddot{\boldsymbol{u}}) \cdot \delta \boldsymbol{u} \mathrm{d} V+\int_{S^{t}}(\hat{\boldsymbol{t}}-\boldsymbol{n} \cdot \boldsymbol{\sigma}) \cdot \delta \boldsymbol{u} \mathrm{d} S+\int_{S^{u}}(\hat{\boldsymbol{u}}-\boldsymbol{u}) \cdot \delta \boldsymbol{t} \mathrm{d} S\right) \mathrm{d} t=0
$$


Here $\boldsymbol{\sigma}$ is the stress, $\boldsymbol{f}$ is the volume force and $\boldsymbol{n}$ is the unit normal vector. A prescribed traction $\hat{\boldsymbol{t}}$ acts on a subsurface denoted by $S^{t}$ and a prescribed displacement $\hat{\boldsymbol{u}}$ acts on the complementary surfaces $S^{u}$. Since the virtual displacement components in $\delta \boldsymbol{u}$ and the virtual traction components $\delta \boldsymbol{t}$ are independent, equation (1) reduces to separate equations for each variational term. For sake of clarity, each equation is written below on component form. The equations of motion contained in the volume integrals are thus

$$
\begin{aligned}
& \int_{V}\left(\frac{\partial \sigma_{r r}}{\partial r}+\frac{1}{r} \frac{\partial \sigma_{r \theta}}{\partial \theta}+\frac{\partial \sigma_{r z}}{\partial z}+\frac{\sigma_{r r}-\sigma_{\theta \theta}}{r}+\rho f_{r}-\rho \frac{\partial^{2} u}{\partial t^{2}}\right) \delta u r \mathrm{~d} r \mathrm{~d} \theta \mathrm{d} z=0 \\
& \int_{V}\left(\frac{\partial \sigma_{r \theta}}{\partial r}+\frac{1}{r} \frac{\partial \sigma_{\theta \theta}}{\partial \theta}+\frac{\partial \sigma_{\theta z}}{\partial z}+2 \frac{\sigma_{r \theta}}{r}+\rho f_{\theta}-\rho \frac{\partial^{2} v}{\partial t^{2}}\right) \delta v r \mathrm{~d} r \mathrm{~d} \theta \mathrm{d} z=0 \\
& \int_{V}\left(\frac{\partial \sigma_{r z}}{\partial r}+\frac{1}{r} \frac{\partial \sigma_{\theta z}}{\partial \theta}+\frac{\partial \sigma_{z z}}{\partial z}+\frac{\sigma_{r z}}{r}+\rho f_{z}-\rho \frac{\partial^{2} w}{\partial t^{2}}\right) \delta w r \mathrm{~d} r \mathrm{~d} \theta \mathrm{d} z=0
\end{aligned}
$$

The surface integrals at the lateral surface $r=a$ become

$$
\begin{aligned}
& \int_{S_{a, r}^{t}}\left(\hat{t}_{r}-\sigma_{r r}\right) \delta u \mathrm{~d} \theta \mathrm{d} z=\int_{S_{a, \theta}^{t}}\left(\hat{t}_{\theta}-\sigma_{r \theta}\right) \delta v \mathrm{~d} \theta \mathrm{d} z=\int_{S_{a, z}^{t}}\left(\hat{t}_{z}-\sigma_{r z}\right) \delta w \mathrm{~d} \theta \mathrm{d} z=0, \\
& \int_{S_{a, r}^{u}}(\hat{u}-u) \delta t_{r} \mathrm{~d} \theta \mathrm{d} z=\int_{S_{a, \theta}^{u}}(\hat{v}-v) \delta t_{\theta} \mathrm{d} \theta \mathrm{d} z=\int_{S_{a, z}^{u}}(\hat{w}-w) \delta t_{w} \mathrm{~d} \theta \mathrm{d} z=0
\end{aligned}
$$

where $\hat{t}_{r}$ is prescribed on $S_{a, r}^{t}$ etc. The surface integrals on the end surfaces $z=\{0, L\}$ are written

$$
\begin{aligned}
& \int_{S_{\{0, L\}, r}^{t}}\left(\hat{t}_{r} \pm \sigma_{r z}\right) \delta u r \mathrm{~d} r \mathrm{~d} \theta=\int_{S_{\{0, L\}, \theta}^{t}}\left(\hat{t}_{\theta} \pm \sigma_{\theta z}\right) \delta v r \mathrm{~d} r \mathrm{~d} \theta=\int_{S_{\{0, L\}, z}^{t}}\left(\hat{t}_{z} \pm \sigma_{z z}\right) \delta w r \mathrm{~d} r \mathrm{~d} \theta=0, \\
& \int_{S_{\{0, L\}, r}^{u}}(\hat{u}-u) \delta t_{r} r \mathrm{~d} r \mathrm{~d} \theta=\int_{S_{\{0, L\}, \theta}^{u}}(\hat{v}-v) \delta t_{\theta} r \mathrm{~d} r \mathrm{~d} \theta=\int_{S_{\{0, L\}, z}^{u}}(\hat{w}-w) \delta t_{w} r \mathrm{~d} r \mathrm{~d} \theta=0,
\end{aligned}
$$

where $\hat{t}_{r}$ is prescribed on $S_{\{0, L\}, r}^{t}$ etc. The notation \pm in (7) refers to the left/right end.

These set of three dimensional equations with pertinent boundary conditions constitute the base in order to systematically develop approximate cylinder equations. As will be described below (2)-(4) results in recursion formulas, (5)-(6) results in a hierarchy of equations of motion while (7)-(8) results in a hierarchy of end boundary conditions. 


\section{Series expansion}

In order to derive sets of approximate cylinder equations, the displacement fields are to be expanded in power series in the radial coordinate $r[30,31]$

$$
\begin{aligned}
& u=u_{0}(\theta, z, t)+r u_{1}(\theta, z, t)+r^{2} u_{2}(\theta, z, t)+\ldots \\
& v=v_{0}(\theta, z, t)+r v_{1}(\theta, z, t)+r^{2} v_{2}(\theta, z, t)+\ldots \\
& w=w_{0}(\theta, z, t)+r w_{1}(\theta, z, t)+r^{2} w_{2}(\theta, z, t)+\ldots
\end{aligned}
$$

By using this ansatz in the stress-displacement relations

$$
\begin{aligned}
& \sigma_{r r}=\lambda\left[\frac{1}{r} \frac{\partial}{\partial r}(r u)+\frac{1}{r} \frac{\partial v}{\partial \theta}+\frac{\partial w}{\partial z}\right]+2 \mu \frac{\partial u}{\partial r}, \\
& \sigma_{\theta \theta}=\lambda\left[\frac{1}{r} \frac{\partial}{\partial r}(r u)+\frac{1}{r} \frac{\partial v}{\partial \theta}+\frac{\partial w}{\partial z}\right]+2 \mu\left[\frac{u}{r}+\frac{1}{r} \frac{\partial v}{\partial \theta}\right], \\
& \sigma_{z z}=\lambda\left[\frac{1}{r} \frac{\partial}{\partial r}(r u)+\frac{1}{r} \frac{\partial v}{\partial \theta}+\frac{\partial w}{\partial z}\right]+2 \mu \frac{\partial w}{\partial z}, \\
& \sigma_{r \theta}=\mu\left[\frac{1}{r} \frac{\partial u}{\partial \theta}+\frac{\partial v}{\partial r}-\frac{v}{r}\right], \quad \sigma_{r z}=\mu\left[\frac{\partial u}{\partial z}+\frac{\partial w}{\partial r}\right], \quad \sigma_{\theta z}=\mu\left[\frac{\partial v}{\partial z}+\frac{1}{r} \frac{\partial w}{\partial \theta}\right],
\end{aligned}
$$

the stress expressions are also written on series form

$$
\sigma_{i j}=r^{-1} \sigma_{i j,-1}(\theta, z, t)+\sigma_{i j, 0}(\theta, z, t)+r \sigma_{i j, 1}(\theta, z, t)+\ldots
$$

which thus may be used in (2)-(4). Considering (2), the expression in the parenthesis is readily written as $\mathcal{F}_{r}=r^{-2} \mathcal{F}_{r,-2}(\theta, z, t)+r^{-1} \mathcal{F}_{r,-1}(\theta, z, t)+\ldots$. This implies that $(2)$ holds provided that

$$
\int_{0}^{a}\left(r^{-2} \mathcal{F}_{r,-2}+r^{-1} \mathcal{F}_{r,-1}+\ldots\right)\left(\delta u_{0}+r \delta u_{1}+\ldots\right) r \mathrm{~d} r=0
$$

By performing the radial integrations, and using that the virtual displacements $\delta u_{k}$ are independent, a unique solution is obtained when $\mathcal{F}_{r, k-2}=0$ for $k=0,1,2, \ldots$. Similar arguments hold for $\mathcal{F}_{\theta}$ and $\mathcal{F}_{z}$ in (3) and (4). In the case of no volume forces $\boldsymbol{f}=\boldsymbol{O}$, these 
relations are explicitly given as

$$
\begin{aligned}
& \mathcal{F}_{r, k-2}=\left(k^{2}-1\right)(\lambda+2 \mu) u_{k}+\mu \frac{\partial^{2} u_{k}}{\partial \theta^{2}}+((k-1) \lambda+(k-3) \mu) \frac{\partial v_{k}}{\partial \theta} \\
& +(k-1)(\lambda+\mu) \frac{\partial w_{k-1}}{\partial z}+\mu \frac{\partial^{2} u_{k-2}}{\partial z^{2}}-\rho \frac{\partial^{2} u_{k-2}}{\partial t^{2}}=0 \\
& \mathcal{F}_{\theta, k-2}=\left(k^{2}-1\right) \mu v_{k}+(\lambda+2 \mu) \frac{\partial^{2} v_{k}}{\partial \theta^{2}}+((k+1) \lambda+(k+3) \mu) \frac{\partial u_{k}}{\partial \theta} \\
& +(\lambda+\mu) \frac{\partial^{2} w_{k-1}}{\partial \theta \partial z}+\mu \frac{\partial^{2} v_{k-2}}{\partial z^{2}}-\rho \frac{\partial^{2} v_{k-2}}{\partial t^{2}}=0, \\
& \mathcal{F}_{z, k-2}=k^{2} \mu w_{k}+\mu \frac{\partial^{2} w_{k}}{\partial \theta^{2}}+k(\lambda+\mu) \frac{\partial u_{k-1}}{\partial z} \\
& +(\lambda+\mu) \frac{\partial^{2} v_{k-1}}{\partial \theta \partial z}+(\lambda+2 \mu) \frac{\partial^{2} w_{k-2}}{\partial z^{2}}-\rho \frac{\partial^{2} w_{k-2}}{\partial t^{2}}=0
\end{aligned}
$$

for $k=0,1,2, \ldots$ Here $\left\{u_{k}, v_{k}, w_{k}\right\} \equiv 0$ for $k<0$. These equations are recursion formulas, that are exact provided that the displacement fields may be expanded in an infinite power series (9). Similar results are presented by Hägglund and Folkow [33] for shells using a more direct method not based on Hamilton's principle, where (13)-(15) may be obtained as the limit when the shell radius tends to zero (there are a few sign typos in [33]). Contrary to the rod, plate, and shell cases $[31,32,33]$, the highest order fields $\left\{u_{k}, v_{k}, w_{k}\right\}$ in (13)-(15) may not directly be extracted and expressed on non-derivative form in terms of the lower order recursion fields $\left\{u_{k-1}, v_{k-1}, \ldots\right\}$ due to the derivatives with respect to the circumferential coordinate in the highest order terms. In order to resolve this matter, the traditional method of expanding the fields in Fourier series with respect to the circumferential coordinate is introduced. Hereby the recursion relations, the boundary conditions etc. may all be handled separately for each Fourier mode.

\section{Fourier expansion}

Each term in the radial series ansatz (9) are expanded in Fourier series according to

$u_{k}=\sum_{m=0}^{\infty} u_{k, m}(z, t) \cos m \theta, \quad v_{k}=\sum_{m=0}^{\infty} v_{k, m}(z, t) \sin m \theta, \quad w_{k}=\sum_{m=0}^{\infty} w_{k, m}(z, t) \cos m \theta$.

Here the angle $\theta$ is measured from a vertical axis in a plane through the cross section of the cylinder with a horizontal axis, as defined in [39]. Hereby, the case $m=1$ correspond to the flexural motion in the vertical direction. The axisymmetric case $m=0$ is for a rod with radial and longitudinal motion, treated in [30,31]. The torsional case obtained by interchanging $\cos m \theta$ and $\sin m \theta$ in (16) for $m=0$ is studied separately in Section 6 .

Adopting (16) in the recursion relations (13)-(15) results in separate equations for each 
Fourier mode $m$. By studying these equations systematically for each $k$ starting from the lowest circumferential mode, it appears that $u_{k, m}$ and $v_{k, m}$ are zero when $k$ and $m$ are either both even or both odd, respectively. The opposite situation holds for $w_{k, m}$. Moreover,

$$
u_{k, m}=v_{k, m}=w_{k, m-1} \equiv 0, \quad k<m-1 .
$$

Hence, the upper limit for the sums in (16) are hereby finite. In other words, the radial expansion for each higher circumferential mode starts from a higher radial power. Hereby, equation (16) inserted in (9) can be collected as

$$
u=\sum_{m=0}^{\infty} \tilde{u}_{m}(r, z, t) \cos m \theta, \quad v=\sum_{m=0}^{\infty} \tilde{v}_{m}(r, z, t) \sin m \theta, \quad w=\sum_{m=0}^{\infty} \tilde{w}_{m}(r, z, t) \cos m \theta
$$

where

$$
\begin{aligned}
& \tilde{u}_{m}=r^{m-1} u_{m-1, m}+r^{m+1} u_{m+1, m}+r^{m+3} u_{m+3, m}+\ldots, \\
& \tilde{v}_{m}=r^{m-1} v_{m-1, m}+r^{m+1} v_{m+1, m}+r^{m+3} v_{m+3, m}+\ldots, \\
& \tilde{w}_{m}=r^{m} w_{m, m}+r^{m+2} w_{m+2, m}+r^{m+4} w_{m+4, m}+\ldots
\end{aligned}
$$

Note again that $u_{m-1, m}=v_{m-1, m} \equiv 0$ for $m=0$. The explicit expressions for the nonzero terms in the recursion relations become from (13)-(15)

$$
\begin{aligned}
& (k+1)(k+3)\left[(k+1)(k+3)+4 m(k+2)+4 m^{2}\right] \mu(\lambda+2 \mu) u_{m+k+2, m}= \\
& {\left[(m+k+1)(m+k+3) \mu-m^{2}(\lambda+2 \mu)\right]\left(\rho \ddot{u}_{m+k, m}-\mu u_{m+k, m}^{\prime \prime}\right)} \\
& -[m(m+k+1)(\lambda+\mu)-2 m \mu]\left(\rho \ddot{v}_{m+k, m}-\mu v_{m+k, m}^{\prime \prime}\right) \\
& -(k+1)(m+k+3)(2 m+k+1) \mu(\lambda+\mu) w_{m+k+1, m}^{\prime}, \quad k=1,3, \ldots, \\
& (k+1)(k+3)\left[(k+1)(k+3)+4 m(k+2)+4 m^{2}\right] \mu(\lambda+2 \mu) v_{m+k+2, m}= \\
& {\left[(m+k+1)(m+k+3)(\lambda+2 \mu)-m^{2} \mu\right]\left(\rho \ddot{v}_{m+k, m}-\mu v_{m+k, m}^{\prime \prime}\right)} \\
& +[m(m+k+3)(\lambda+\mu)+2 m \mu]\left(\rho \ddot{u}_{m+k, m}-\mu u_{m+k, m}^{\prime \prime}\right) \\
& +m(k+1)(2 m+k+1) \mu(\lambda+\mu) w_{m+k+1, m}^{\prime}, \\
& {\left[(m+k+2)^{2}-m^{2}\right] \mu w_{m+k+2, m}=\rho \ddot{w}_{m+k, m}-(\lambda+2 \mu) w_{m+k, m}^{\prime \prime}} \\
& -(m+k+2)(\lambda+\mu) u_{m+k+1, m}^{\prime}-m(\lambda+\mu) v_{m+k+1, m}^{\prime}, \quad k=1,3, \ldots,
\end{aligned}
$$

Here a prime denotes a $z$-derivative. Equations (20) and (21) are obtained by a combination of (13) and (14). Two further equations are also be obtained by combining the recursion 
relations for negative $k$ values

$$
\begin{aligned}
& u_{m-1, m}+v_{m-1, m}=0 \\
& {\left[m(m+2)(\lambda+2 \mu)-m^{2} \mu\right] u_{m+1, m}+\left[m^{2}(\lambda+\mu)-2 m \mu\right] v_{m+1, m}} \\
& =\rho \ddot{u}_{m-1, m}-\mu u_{m-1, m}^{\prime \prime}-m(\lambda+\mu) w_{m, m}^{\prime}
\end{aligned}
$$

Equations (23) and (24) provide constraint relations between $u_{m-1, m}$ and $v_{m-1, m}$, as well as between $u_{m+1, m}, v_{m+1, m}$ and $w_{m, m}$, respectively. The recursion formulas (20)-(22) together with (23) and (24) allow for expressing higher order index terms in the mutually independent lowest order index terms. This is to be used in the derivation process for obtaining a hierarchy of approximate cylinder equations with pertinent boundary conditions. The natural choice would be to use the lowest index non-zero terms in line with earlier work $[31,32,33]$; that is $u_{m-1, m}, v_{m-1, m}$ and $w_{m, m}$ for $m>0$. However, due to the constraint (23) another set must be opted for which here is set to be $u_{m-1, m}, v_{m+1, m}$ and $w_{m, m}$ for $m>0$. In the special case $m=0$, the fields are expressed in $u_{1,0}, v_{1,0}$, and $w_{0,0}$ as there is no constraint in that case.

The expressions for the stresses follow directly from (11) using (18). Hereby $\left\{\sigma_{r r}, \sigma_{\theta \theta}, \sigma_{z z}, \sigma_{r z}\right\}$ are expanded in $\cos m \theta$ and $\left\{\sigma_{r \theta}, \sigma_{\theta z}\right\}$ are expanded in $\sin m \theta$. The stresses may be written

$$
\sigma_{i j}=\sum_{m=0}^{\infty} \tilde{\sigma}_{i j, m}(r, z, t)\{\cos m \theta ; \sin m \theta\}
$$

using either $\cos m \theta$ or $\sin m \theta$ according to above. The Fourier modes are

$$
\begin{aligned}
& \tilde{\sigma}_{a b, m}=r^{m-2} \sigma_{a b,\{m-2, m\}}+r^{m} \sigma_{a b,\{m, m\}}+r^{m+2} \sigma_{a b,\{m+2, m\}}+\ldots, \\
& \tilde{\sigma}_{c d, m}=r^{m-1} \sigma_{c d,\{m-1, m\}}+r^{m+1} \sigma_{c d,\{m+1, m\}}+r^{m+3} \sigma_{c d,\{m+3, m\}}+\ldots,
\end{aligned}
$$

where $a b$ is for $\{r r, \theta \theta, z z, r \theta\}$ and $c d$ is for $\{r z, \theta z\}$. Each stress term is expressed as

$$
\begin{aligned}
& \sigma_{r r,\{k, m\}}(z, t)=[(k+2)(\lambda+2 \mu)-2 \mu] u_{k+1, m}+m \lambda v_{k+1, m}+\lambda w_{k, m}^{\prime}, \\
& \sigma_{\theta \theta,\{k, m\}}(z, t)=[(k+2) \lambda+2 \mu] u_{k+1, m}+m(\lambda+2 \mu) v_{k+1, m}+\lambda w_{k, m}^{\prime}, \\
& \sigma_{z z,\{k, m\}}(z, t)=(k+2) \lambda u_{k+1, m}+m \lambda v_{k+1, m}+(\lambda+2 \mu) w_{k, m}^{\prime}, \\
& \sigma_{r \theta,\{k, m\}}(z, t)=\mu\left[k v_{k+1, m}-m u_{k+1, m}\right], \\
& \sigma_{r z,\{k, m\}}(z, t)=\mu\left[u_{k, m}^{\prime}+(k+1) w_{k+1, m}\right], \\
& \sigma_{\theta z,\{k, m\}}(z, t)=\mu\left[v_{k, m}^{\prime}-m w_{k+1, m}\right] .
\end{aligned}
$$

Note from (29) that $\sigma_{z z,\{m-2, m\}}=0$ due to (17) and (23). Similarly, $\sigma_{r r,\{m-2, m\}}=\sigma_{\theta \theta,\{m-2, m\}}=$ $\sigma_{r \theta,\{m-2, m\}}=0$ for the special cases $m=0$ and $m=1$, while $\sigma_{r z,\{m-1, m\}}=\sigma_{\theta z,\{m-1, m\}}=0$ for $m=0$. Hereby no negative powers of $r$ appear in the stresses (26). 


\section{Boundary conditions}

\subsection{Lateral boundary conditions; Equations of motion}

The lateral boundary conditions (BCs) at $r=a$ are obtained through (5) and (6). Consider the first relation in (5) for a prescribed traction $\hat{t}_{r}$ given on a circular ring at fixed $z_{0}$. Using (18) and (25) gives

$$
\int_{0}^{2 \pi}\left(\hat{t}_{r}\left(\theta, z_{0}, t\right)-\sum_{m=0}^{\infty} \tilde{\sigma}_{r r, m}\left(a, z_{0}, t\right) \cos m \theta\right)\left(\sum_{n=0}^{\infty} \delta \tilde{u}_{n}\left(a, z_{0}, t\right) \cos n \theta\right) \mathrm{d} \theta=0 .
$$

Since (33) should hold for each virtual displacement $\delta \tilde{u}_{n}$, orthogonality effects imply the expected result that the equation is fulfilled provided that $\tilde{\sigma}_{r r, m}\left(a, z_{0}, t\right)=\hat{t}_{r, m}\left(z_{0}, t\right)$ where

$$
\hat{t}_{r}\left(\theta, z_{0}, t\right)=\sum_{m=0}^{\infty} \hat{t}_{r, m}\left(z_{0}, t\right) \cos m \theta
$$

Similar arguments hold for the other relations in (5) and (6). The Fourier modes of prescribed lateral tractions are then equal to the corresponding stress modes (26) using (27), (30) or (31). Similarly, the Fourier modes of prescribed lateral displacements are equal to the corresponding displacement modes (19). The latter case with prescribed displacements becomes

$$
\begin{aligned}
& a^{m-1} u_{m-1, m}+a^{m+1} u_{m+1, m}+a^{m+3} u_{m+3, m}+\ldots=\hat{u}_{m}, \\
& a^{m-1} v_{m-1, m}+a^{m+1} v_{m+1, m}+a^{m+3} v_{m+3, m}+\ldots=\hat{v}_{m}, \\
& a^{m} w_{m, m}+a^{m+2} w_{m+2, m}+a^{m+4} w_{m+4, m}+\ldots=\hat{w}_{m},
\end{aligned}
$$

while the former case with prescribed tractions results in

$$
\begin{aligned}
& a^{m-2}\left[(m(\lambda+2 \mu)-2 \mu) u_{m-1, m}+m \lambda v_{m-1, m}\right]+ \\
& a^{m}\left[((m+2)(\lambda+2 \mu)-2 \mu) u_{m+1, m}+m \lambda v_{m+1, m}+\lambda w_{m, m}^{\prime}\right]+\ldots=\hat{t}_{r, m}, \\
& a^{m-2} \mu\left[(m-2) v_{m-1, m}-m u_{m-1, m}\right]+a^{m} \mu\left[m v_{m+1, m}-m u_{m+1, m}\right]+\ldots=\hat{t}_{\theta, m} \\
& a^{m-1} \mu\left[u_{m-1, m}^{\prime}+m w_{m, m}\right]+a^{m+1} \mu\left[u_{m+1, m}^{\prime}+(m+2) w_{m+2, m}\right]+\ldots=\hat{t}_{z, m} .
\end{aligned}
$$

These lateral BCs (35)-(40) constitute the sought set of approximate cylinder equations of motion. Hence, by stating one of the fields for each of the three pairs $\left\{\hat{t}_{r}, \hat{u}\right\},\left\{\hat{t}_{\theta}, \hat{v}\right\},\left\{\hat{t}_{z}, \hat{w}\right\}$ along the lateral surface, a set of three equations forming a hierarchy of approximate cylinder equations is obtained. This system may be truncated to any order. Adopting the recursion relations (20)-(22) together with (23) and (24), these cylinder equations are expressed as a set of hyperbolic partial differential equations in terms of the mutually 
independent lowest order index terms. As noted before, the independent terms for $m=0$ are $u_{1,0}, v_{1,0}$, and $w_{0,0}$. Here, the longitudinal case adopts $u_{1,0}$ and $w_{0,0}$ in the appropriate pair of equations from (35), (37), (38), and (40), while the torsional case uses $v_{1,0}$ in either (36) or (39). For the flexural modes $m>0$ one has $u_{m-1, m}, v_{m+1, m}$ and $w_{m, m}$ as independent terms, using the full set of three equations. Since the longitudinal case has been studied previously $[30,31]$, the torsional and flexural motions are here of most interest.

For simplicity, consider the standard case of only prescribed tractions (38)-(40), noting that the displacement based lateral boundary conditions (35)-(37) or mixed boundary conditions may be treated in a similar manner. Now, the first terms with negative power of $a$ (for $m=0$ and $m=1$ ) are seen to be zero due to the power series ansatz (9) and the constraint (23). Consider next the flexural case $(m>0)$ more in detail. For $n$ terms in (38) and (39) (including the zero terms), the highest power of $a$ is $m+2 n-4$ which involves the terms $u_{m+2 n-3, m}$ and $v_{m+2 n-3, m}$. By using the recursion relation (20) and (23)-(24), equation (38) involves $2 n-2$ order time and spatial derivatives of the radial displacement $u_{m-1, m}$. Similarly, the recursion relation (21) and (23)-(24) imply that (39) involves $2 n-4$ $(n>1)$ order time and spatial derivatives of the circumferential displacement $v_{m+1, m}$. In the same fashion when using $n$ terms in (40), the highest power of $a$ is $m+2 n-3$ which involves the longitudinal displacement terms $w_{m+2 n-2, m}$. Adopting the recursion relations (22) and (23)-(24) give that (40) involves $2 n-2$ order time and spatial derivatives of $w_{m, m}$.

So, the resulting hyperbolic equations for flexural motion $(m>0)$ using $n_{r}$ terms in (38), $n_{\theta}$ terms in (39) and $n_{z}$ terms in (40) are of total differential order $2\left(n_{r}+n_{\theta}+n_{z}\right)-8$ in both space and time. This is readily seen by eliminating within the set of equations, so as to obtain one equation in one of the fields, say $u_{m-1, m}$. Due to the same differential terms appearing in both (38) and (39), it is natural to set $n_{\theta}=n_{r}$. As for (40), the level of higher order derivatives implies that one should choose $n_{z}=n_{r}$ or $n_{z}=n_{r}-1$. For nontrivial solutions, (38)-(40) are solved using $n_{i}>1$. For the flexural case $m=1$, the first $a^{-1}$ terms in (38) and (39) are zero which calls for $n_{r}>2$ and $n_{\theta}>2$.

\subsection{End boundary conditions}

The end BCs at $z=\{0, L\}$ are obtained through (7) and (8). Consider for example the last generalized force equilibrium relation in (7) for a prescribed traction $\hat{t}_{z}$ given on $z=L$. For the circumferential coordinate, similar relations as in (33) are obtained, resulting in $\tilde{\sigma}_{z z, m}(r, L, t)=\hat{t}_{z, m}(r, t)$ where $\hat{t}_{z, m}(r, t)$ is the Fourier expansion mode of the prescribed traction as in (34). As for the radial dependence for fixed $m,(7)$ becomes from the expansions (19) and (26)

$$
\int_{0}^{a}\left(\hat{t}_{z, m}(r, t)-\sum_{i=0}^{\infty} r^{m+2 i} \sigma_{z z,\{m+2 i, m\}}(L, t)\right)\left(\sum_{j=0}^{\infty} r^{m+2 j} \delta w_{m+2 j, m}(L, t)\right) r \mathrm{~d} r=0,
$$


noting that $\sigma_{z z,\{m-2, m\}}=0$. By performing the radial integration for each virtual displacement $\delta w_{m+2 j, m}$, the resulting equation system may be solved for every $\sigma_{z z,\{m+2 i, m\}}$ term. In other words, assuming truncation order $N$ gives

$$
\int_{0}^{a}\left(\hat{t}_{z, m}(r, t)-\sum_{i=0}^{N} r^{m+2 i} \sigma_{z z,\{m+2 i, m\}}(L, t)\right) r^{m+1+2 j} \mathrm{~d} r=0, \quad j=0,1, \cdots, N,
$$

where it is straightforward to derive the $N+1$ fields $\sigma_{z z,\{m+2 i, m\}}$ from the $N+1$ integrals (42). The solution is seen to be equivalent to expanding the prescribed traction in orthogonal Zernike polynomials $R_{m+2 i}^{m}(r / a)$ [40]

$$
\hat{t}_{z, m}(r, t) \approx \sum_{i=0}^{N} \hat{t}_{z R,\{2 i, m\}}(t) R_{m+2 i}^{m}(r / a)=\sum_{i=0}^{N} \hat{t}_{z,\{2 i, m\}}(t) r^{m+2 i}
$$

where thus $\sigma_{z z,\{m+2 i, m\}}=\hat{t}_{z,\{2 i, m\}}$ for $i=0,1, \cdots, N$. Note that the standard case $\hat{t}_{z, m}(r, t)=$ 0 results in $\sigma_{z z,\{m+2 i, m\}}=0$ for all $i$. In a similar manner for prescribed shear tractions $\hat{t}_{r}$ and $\hat{t}_{\theta}$ given on $z=L$, the Zernike polynomials are used as

$\hat{t}_{j, 0}(r, t)=\sum_{i=0}^{\infty} \hat{t}_{j R,\{2 i, 0\}}(t) R_{1+2 i}^{1}(r / a), \quad \hat{t}_{j, m}(r, t)=\sum_{i=0}^{\infty} \hat{t}_{j R,\{2 i, m\}}(t) R_{m-1+2 i}^{m-1}(r / a), \quad m>0$

where $j=\{r, \theta\}$. Prescribed displacements (8) are treated in an analogous way. Hence, for each point at the end surfaces, one of the fields for each of the three pairs $\left\{\hat{t}_{r}, \hat{u}\right\},\left\{\hat{t}_{\theta}, \hat{v}\right\},\left\{\hat{t}_{z}, \hat{w}\right\}$ is to be given.

For the truncated cylinder equation set of total differential order $2\left(n_{r}+n_{\theta}+n_{z}\right)-8$ discussed above when $m>0$, there are $n_{r}+n_{\theta}+n_{z}-4$ boundary conditions at each end to be stated for a fixed $m$. In line with the discussion on lateral BCs above, it is natural to assume $n_{r}-1$ BCs in the radial direction and $n_{\theta}-1$ BCs in the circumferential direction at each end. Consequently, for prescribed shear stresses, the sets $\left\{\sigma_{r z,\{m-1, m\}}, \ldots, \sigma_{r z,\left\{m+2 n_{r}-5, m\right\}}\right\}$ and $\left\{\sigma_{\theta z,\{m-1, m\}}, \ldots, \sigma_{\theta z,\left\{m+2 n_{\theta}-5, m\right\}}\right\}$ from (26) and (31)-(32) are to be given by means of Zernike expansion of $\hat{t}_{r, m}(r, t)$ with $n_{r}-1$ terms and $\hat{t}_{\theta, m}(r, t)$ with $n_{\theta}-1$ terms, respectively. In the same fashion for prescribed displacements, the sets $\left\{u_{m-1, m}, \ldots, u_{m+2 n_{r}-5, m}\right\}$ and $\left\{v_{m-1, m}, \ldots, v_{m+2 n_{\theta}-5, m}\right\}$ from (19) are prescribed using Zernike polynomials. At first sight this seems to result in an inadequate number of boundary conditions in the normal direction. However, due to the constraint (23) the first displacement terms $u_{m-1, m}$ and $v_{m-1, m}$ render the same $\mathrm{BC}$ as do the corresponding stress expressions for $\sigma_{r z,\{m-1, m\}}$ and $\sigma_{\theta z,\{m-1, m\}}$. Hereby the total number of shear BCs are reduced by one, resulting in $n_{r}+n_{\theta}-3$ at each end. Consequently there are $n_{z}-1 \mathrm{BCs}$ at each end in the normal direction as expected. For a prescribed stress using (26) and (29) this results in the set $\left\{\sigma_{z z,\{m, m\}}, \ldots, \sigma_{z z,\left\{m+2 n_{z}-4, m\right\}}\right\}$, while a prescribed displacement gives $\left\{w_{m, m}, \ldots, w_{m+2 n_{z}-4, m}\right\}$ from (19). 
These sets of end boundary conditions may then be expressed in terms of the lowest order expansion fields $u_{m-1, m}, v_{m+1, m}$ and $w_{m, m}$ adopting the recursion relations (20)-(24). The highest spatial derivatives of the boundary expansion fields are hereby one order less for the prescribed stress expressions, and two order less for the prescribed displacement expressions, when compared to the various sets of equations of motion (35)-(40).

\section{Torsional mode, $m=0$}

So far all equations are given on a quite general form, covering all mode families. Using recursion equations it is possible to obtain explicit time domain partial differential cylinder equations in terms of the lowest order displacement terms. This can (in principle) be done to arbitrary order. Among the mode families (longitudinal, torsional, flexural), the torsional case $(m=0)$ is the simplest to handle and is presented on a compact form below. The longitudinal case $(m=0)$ has been studied previously [30] while the various flexural families $(m>0)$ results in more extensive partial differential equations which may not easily be written on a general form.

Consider the torsional mode where the fields $v$ do not couple to $u$ and $w$. By using (36) for prescribed lateral displacement, or (39) for prescribed lateral traction, this gives for $N$ terms in each case

$$
\begin{aligned}
& \sum_{k=0}^{N-1} a^{2 k+1} v_{2 k+1,0}+\mathcal{O}\left(a^{2 N+1}\right)=\hat{v}_{0}, \\
& \sum_{k=0}^{N-1} 2 \mu(k+1) a^{2(k+1)} v_{2 k+3,0}+\mathcal{O}\left(a^{2(N+1)}\right)=\hat{t}_{\theta, 0} .
\end{aligned}
$$

The recursion formula (21) results in

$$
v_{k+2,0}=\frac{1}{(k+1)(k+3) \mu}\left(\rho \ddot{v}_{k, 0}-\mu v_{k, 0}^{\prime \prime}\right), \quad \quad k=1,3, \ldots
$$

By using (46) repeatedly, the higher order terms may be expressed in $v_{1,0}$ as

$$
v_{2 k+3,0}=\frac{1}{4^{k+1}(k+1) !(k+2) !}\left(\frac{1}{c_{T}^{2}} \frac{\partial^{2}}{\partial t^{2}}-\frac{\partial^{2}}{\partial z^{2}}\right)^{k+1} v_{1,0}, \quad k=0,1,2, \ldots
$$

where $c_{T}^{2}=\mu / \rho$. Consequently, by using (47) in (45) gives the final equations for the 
torsional mode in terms of the lowest order field

$$
\begin{aligned}
& \sum_{k=0}^{N-1} \frac{a}{k !(k+1) !}\left[\frac{a^{2}}{4}\left(\frac{1}{c_{T}^{2}} \frac{\partial^{2}}{\partial t^{2}}-\frac{\partial^{2}}{\partial z^{2}}\right)\right]^{k} v_{1,0}+\mathcal{O}\left(a^{2 N+1}\right)=\hat{v}_{0}, \\
& \sum_{k=0}^{N-1} \frac{2 \mu}{k !(k+2) !}\left[\frac{a^{2}}{4}\left(\frac{1}{c_{T}^{2}} \frac{\partial^{2}}{\partial t^{2}}-\frac{\partial^{2}}{\partial z^{2}}\right)\right]^{k+1} v_{1,0}+\mathcal{O}\left(a^{2(N+1)}\right)=\hat{t}_{\theta, 0} .
\end{aligned}
$$

Note that taking only the first term in (49) renders the classical wave equation, while taking the first two terms in (48) renders the Klein-Gordon equation.

The end boundary conditions are obtained from truncation of (44). Hence, the prescribed fields at the ends (both for traction $\hat{t}_{\theta}$ and displacement $\hat{v}$ ) are to be expanded in Zernike polynomials $R_{2 k+1}^{1}(r / a)$. Hereby the series terms in (32) and (19) are readily obtained at the boundaries. Note from (32) that for a free end $v_{2 k+1,0}^{\prime}=0$ for all $k$, while from (19) a fixed end gives $v_{2 k+1,0}=0$ for all $k$. The correct end boundary conditions for the simple classical wave equation case, $k=0$ in (49), thus corresponds to $v_{1,0}^{\prime}=0$ for a free end and $v_{1,0}=0$ for a fixed end as expected.

\section{Asymptotic expansions}

This part is to examine whether the proposed series expansion approach render equations that are asymptotically correct. The object is to compare the frequency equations for the present theory to the exact theory. As the longitudinal case $(m=0)$ has been discussed previously [30], the torsional and flexural cases are studied here.

\subsection{Torsional mode, $m=0$}

The torsional equation is rather straightforward to study, being only dependent on the circumferential displacement. By assuming a harmonic wave $v_{1,0}=e^{i\left(k_{z} z-\omega t\right)}$ in (48)-(49), this render in the limit the frequency equations

$$
\begin{array}{lll}
\frac{2 a}{q a} \sum_{k=0}^{\infty} \frac{(-1)^{k}}{k !(k+1) !}\left(\frac{1}{2} q a\right)^{2 k+1}=0 & \Leftrightarrow & 2 J_{1}(q a) / q=0, \\
2 \mu \sum_{k=0}^{\infty} \frac{(-1)^{k}}{k !(k+2) !}\left(\frac{1}{2} q a\right)^{2 k+2}=0, & \Leftrightarrow & 2 \mu J_{2}(q a)=0,
\end{array}
$$

using $q=\sqrt{\omega^{2} / c_{T}^{2}-k_{z}^{2}}$ and $J_{i}$ as the Bessel function of the first kind. The right hand expressions involving Bessel functions are actually the solutions to the exact frequency equations for a fixed lateral boundary (50) and a free lateral boundary (51) [39], noting that 
$J_{2}(q a)=\left(2 J_{1}(q a)-q a J_{0}(q a)\right) / q a$. Hereby, each term using the present series expansion approach is identical to the corresponding Maclaurin expansion term using the exact theory. From this it is concluded that the present series expansion equations are asymptotically correct to arbitrary order.

\subsection{Flexural modes, $m>0$}

The analyzes for the cases $m>0$ are considerably more involved than for the decoupled torsional mode above. For the exact theory the harmonic displacement terms can be stated as

$$
\begin{aligned}
& u=U(r) \cos (m \theta) e^{i\left(k_{z} z-\omega t\right)}, \\
& v=V(r) \sin (m \theta) e^{i\left(k_{z} z-\omega t\right)}, \\
& w=W(r) \cos (m \theta) e^{i\left(k_{z} z-\omega t\right)},
\end{aligned}
$$

where the radial functions are $[1,39]$

$$
\begin{aligned}
& U(r)=A \frac{\partial}{\partial r} J_{m}(p r)+\frac{B}{r} J_{m}(q r)+i k_{z} C J_{m+1}(q r), \\
& V(r)=-\frac{A}{r} J_{m}(p r)-B \frac{\partial}{\partial r} J_{m}(q r)+i k_{z} C J_{m+1}(q r), \\
& W(r)=i k_{z} A J_{m}(p r)-\frac{C}{r} \frac{\partial}{\partial r}\left[r J_{m+1}(q r)\right]-\frac{C}{r} J_{m+1}(q r),
\end{aligned}
$$

using that $p=\sqrt{\omega^{2} / c_{L}^{2}-k_{z}^{2}}$ and $c_{L}$ is the longitudinal phase velocity. In the usual manner, equations (52)-(53) are inserted into a set of three homogeneous lateral boundary conditions, from which the resulting solution constitutes the transcendental frequency equation. By taking a Maclaurin expansion of this equation, it is possible to compare exact theory to the frequency equation from the present series expansion theory. However, contrary to the torsional case, it seems not feasible to obtain general expressions to arbitrary order for the present theory. Studying only the first few terms for an arbitrary $m>0$, the standard case of a free lateral surface render identical terms using the expanded exact frequency equation as for the present theory adopting (38)-(40). The same thing holds for a fixed lateral surface, using (35)-(37) for the present theory. This illustrates that the flexural equations using the present approach is asymptotically correct for these low order terms, and thus probably also to arbitrary order. Similar results are reported for longitudinal displacements in rods [30] and for flexural plates [32, 41] when using a series expansion approach.

In addition to the relations between frequency equations, it is instructive to study a radial Maclaurin series expansion of the Bessel functions in (53). The resulting exact series representation of the displacement fields (52) involve a similar set of non-zero power series terms as presented in (19) for each fixed $m$. Moreover, the recursion relations and 
constraints (20)-(24) also hold for each $m$.

\section{Approximate beam theories, $m=1$}

The various sets of approximate equations for solid cylinders covered in the preceding sections may be truncated to arbitrary order, where higher order sets can be used for obtaining high accuracy solutions, while simpler lower order sets may be used as engineering equations. This latter group of equations are naturally simpler to solve than the higher order sets, and may thus be an alternative to traditional engineering theories. This is especially of interest for the transverse flexural case $m=1$, denoted a beam, where there exist several theories of which the Euler-Bernoulli and the Timoshenko beam equations are frequently used. This section aims at analytically compare the present beam equation to other beam theories presented in the literature. Here only the traditional case of a laterally free beam is studied.

The lowest order set of equations for the present theory that leads to an asymptotically correct beam equation is to include terms of order $a^{2}$ in (38)-(40), that is $n_{r}=n_{\theta}=3$ and $n_{z}=2$. Written out explicitly, the truncated system may be expressed as

$$
\begin{aligned}
& (3 \lambda+4 \mu) u_{2,1}+\lambda v_{2,1}+\lambda w_{1,1}^{\prime}+a^{2}\left[(5 \lambda+8 \mu) u_{4,1}+\lambda v_{4,1}+\lambda w_{3,1}^{\prime}\right]=0, \\
& v_{2,1}-u_{2,1}+a^{2}\left[3 v_{4,1}-u_{4,1}\right]=0 \\
& u_{0,1}^{\prime}+w_{1,1}+a^{2}\left[u_{2,1}^{\prime}+3 w_{3,1}\right]=0 .
\end{aligned}
$$

Using the recursion relations (20)-(22), this is seen to constitute a hyperbolic system of equations. In order to make analytical comparisons to other approximate theories, this set may be reduced to a single equation in terms of one of the fields. The resulting single equation is of 8:th order, and contains all terms up to $a^{2}$, when compared to the nontruncated system. The higher order powers of $a$, that is $a^{4}$ and $a^{6}$, here contain only a part of the total number of terms that would result by including more terms from (38)-(40). So, the truncated single 1D beam equation of order $a^{2}$ is asymptotically correct and reads in terms of $u_{0,1}$ (now denoted $u_{0}$ for simplicity)

$$
\frac{\partial^{2} u_{0}}{\partial t^{2}}+a^{2} \frac{E}{4 \rho}\left(\frac{\partial^{4} u_{0}}{\partial z^{4}}-\alpha \frac{\rho}{E} \frac{\partial^{4} u_{0}}{\partial z^{2} \partial t^{2}}+\beta \frac{\rho^{2}}{E^{2}} \frac{\partial^{4} u_{0}}{\partial t^{4}}\right)+\mathcal{O}\left(a^{4}\right)=0
$$

where $\alpha=(29+8 \nu) / 6$ and $\beta=(1+\nu)\left(16-21 \nu+4 \nu^{2}\right) / 3(1-\nu)$. Here $E$ is the Young's modulus and $\nu$ is the Poisson's ratio.

The present equation could be compared to various classical theories. For the EulerBernoulli (EB) theory, the parameters as written in (55) become $\alpha_{E B}=\beta_{E B}=0$. Considering the Timoshenko (T) equation, the corresponding terms are $\alpha_{T}=(2+2 \nu+\kappa) / \kappa$ and 
$\beta_{T}=2(1+\nu) / \kappa$, where $\kappa$ is the shear correction factor. Of course, none of these two classical theories are truncated as no higher order power terms of $a$ are present. Among more recent higher order theories developed for circular cross sections, Silverman [29] presents a set of beam equations that after elimination to a single equation may be rewritten as a sixth order theory. The terms as in (55) are $\alpha_{S}=(146+101 \nu) / 45$ and $\beta_{S}=101(1+\nu) / 45$, where the $a^{4}$ terms hereby are omitted. Another theory by Kosmatka [27] is applicable on circular cross sections. Here, the general Kosmatka equations may be rewritten as a single eight order equation (including $a^{6}$ terms), while a simplified version turns to a sixth order equation (including $a^{4}$ terms). As the various parameters are only presented numerically in [27] for $\nu=0.333$, it is here instructive to give the parameters $\alpha$ and $\beta$ in (55) numerically for the various beam theories. Table 1 presents the results, where for the Timoshenko theory $\kappa=6(1+\nu)^{2} /\left(7+12 \nu+4 \nu^{2}\right)$ is used [42]. It is clear that the present theory renders higher parameter values than the other theories, especially for the $\beta$ term where the present theory result is roughly twice as big as for the other theories. A similar behavior between different theories is seen for the axisymmetric case [30]. As is discussed in Section 7.2 the expressions for the parameters $\alpha$ and $\beta$ using the present theory are identical to the corresponding $a^{2}$ terms from a series expansion of the exact frequency equation.

\begin{tabular}{cccccc} 
Parameters & $\mathrm{P}$ & $\mathrm{T}$ & $\mathrm{S}$ & $\mathrm{GK}$ & $\mathrm{SK}$ \\
\hline$\alpha$ & 5.277 & 3.861 & 3.992 & 3.980 & 4.142 \\
$\beta$ & 6.296 & 2.861 & 2.992 & 3.104 & 3.142
\end{tabular}

Table 1

The parameters in . (55) for present (P), Timoshenko (T), Silverman (S), general Kosmatka (GK) and simplified Kosmatka (SK) theories, using $\nu=0.333$ and $\kappa=0.932$.

\section{Numerical results}

This section concentrates on numerical results for flexural motion, especially for the lowest transverse case $m=1$. The object is to illustrate the accuracy of the present series expansion approach; both the lower order engineering theories and the higher order sets. The former case is mainly to be compared to traditional engineering theories, while the latter case is to be compared to exact theory. The presented results are for dispersion relation curves for an infinite cylinder, eigenfrequencies for a finite cylinder using three sets of end boundary conditions, as well as mode shapes and stress distributions. These dynamical problems are for laterally free cylinders. Moreover, fix frequency responses due to a prescribed end displacement or a lateral distributed force are also illustrated. In all cases Poisson's ratio is set to $\nu=0.3$. 


\subsection{Dispersion curves}

In order to illustrate the effects from the number of terms adopted in (38)-(40), dispersion relations are calculated for $n_{r}=n_{\theta}=n$ and $n_{z}=n-1$, where $n=3,4,5,6$. A normalized frequency $\Omega=\omega a / c_{E}$ is introduced with $c_{E}^{2}=E / \rho$. Considering the flexural case $m=1$, Figure 1 shows the three lowest modes using both the series expansion theories (38)-(40) and the exact theory involving (52)-(53). It is clear that higher accuracy is obtained as more terms are used. Among the results, the lowest curve is accurately captured in the lower frequency range for all theories. Note that the $n=5$ curve for the second mode virtually coincides with the exact curve, which is also the case for the first mode over most of presented frequencies. Here the case $n=6$ is not plotted as these three curves are indistinguishable from the exact curves in the presented range. Consider next the case

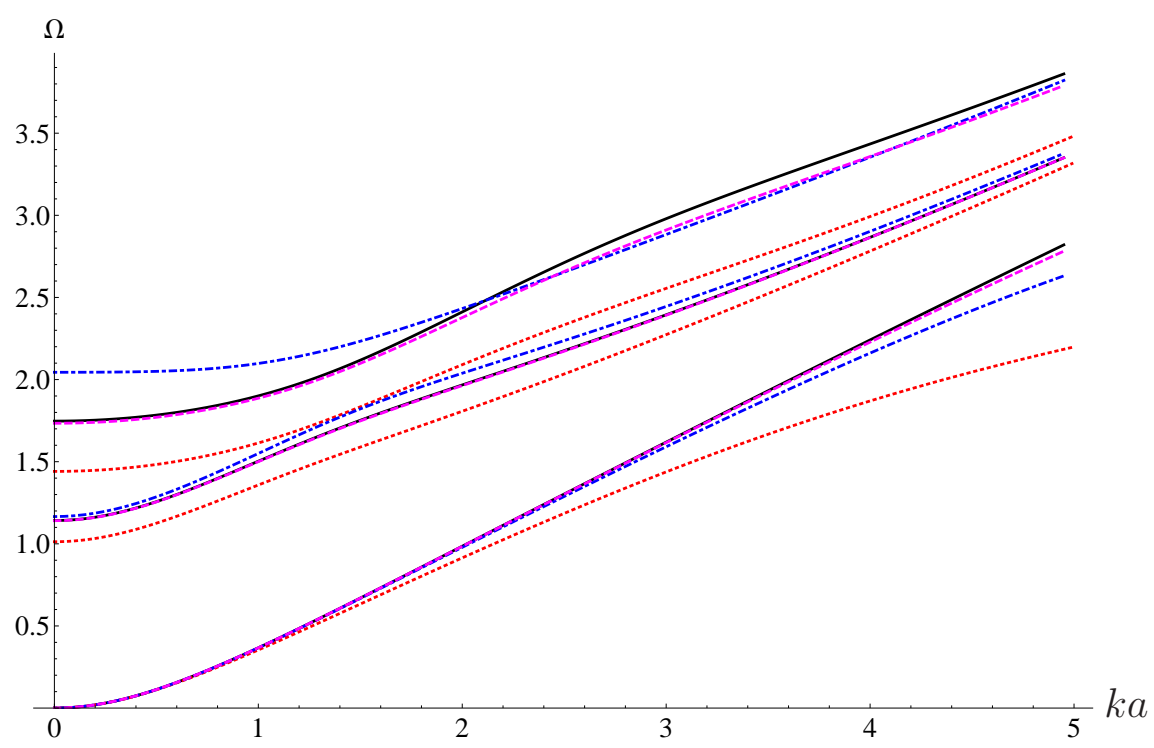

Fig. 1. Dispersion curves for $m=1$ : — Exact, $\cdots n=3, \cdots n=4, \cdots n=5$.

with $m=2$ where the two lowest modes are presented in Figure 2. Here the real part of the second mode for $n=3$ and $n=4$ are plotted as these results are complex valued in this wave number range, except for the interval $0.4<k a<4.0$ in the $n=3$ case. When compared to the $m=1$ case, the first mode is less accurately captured here for the lowest theory $n=3$. This is partly due to the higher frequencies, where the exact cut-off frequency is at $\Omega=1.456$. Similar to the $m=1$ case the $n=5$ curves are close to the exact curves, while the $n=6$ curves (not plotted here) are indistinguishable from the exact curves. The dispersion curves for higher $m$ values could also be displayed in the same fashion, rendering similar results where more terms imply higher accuracy. However, the higher $m$ value the more terms are needed for a specific accuracy.

The present theory of low order may also be compared to classical theories for flexural motion $m=1$. Figure 3 shows the two lowest modes using exact, low order series ex- 


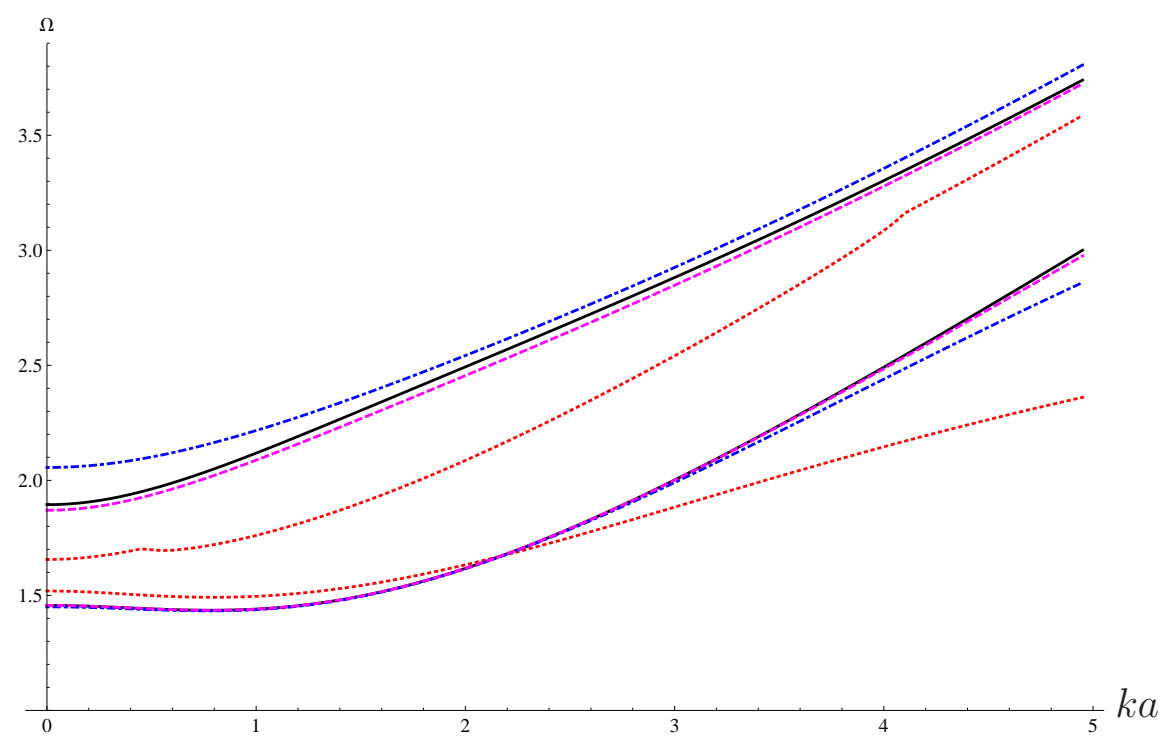

Fig. 2. Dispersion curves for $m=2$ : $—$ Exact, $\cdots n=3,-\cdots n=4,--n=5$.

pansion, Euler-Bernoulli and Timoshenko theories. For the present theory, the truncated single equation of order $a^{2}$ in (55) is here used as an illustration instead of the original set of equations (54) used in Figure 1. As expected the Euler-Bernoulli beam theory is only acceptable at small wave numbers and it progressively deteriorates as the wave number is increased. The Timoshenko beam theory approximates the first dispersion curve very accurately, being virtually indistinguishable from the exact curve at the presented frequencies. This is partly due to the choice of shear coefficient $\kappa$, which is here chosen as in Section 8 so as to render accurate results for long wave lengths [42]. For the second curve the Timoshenko theory behaves well at small wave numbers but the approximation is less accurate as the wave number increases. The present $a^{2}$ order beam theory approximates the first dispersion curve well for small wave numbers, but the approximation deteriorates slightly as the wave number is increased. The cut off frequency for the second dispersion curve is here approximated less accurately than for the Timoshenko theory, although the behavior at higher frequencies is more accurate in the present case. Note the slightly different curves for $n=3$ using (54) presented in Figure 1.

\subsection{Eigenfrequencies}

In this section, the eigenfrequencies for the flexural series expansions theories $m=1$ are compared with one another using different truncation orders. These expansions are also compared to other classical theories as well as the exact theory. Three different end conditions are considered here: simply supported, clamped and free ends. As for the dispersion relations $n_{r}=n_{\theta}=n$ and $n_{z}=n-1$ are chosen. This results in $3 n-5 \mathrm{BCs}$ at each end according to Section 5.2. Of these $2 n-3$ are shear BCs (divided between radial and 


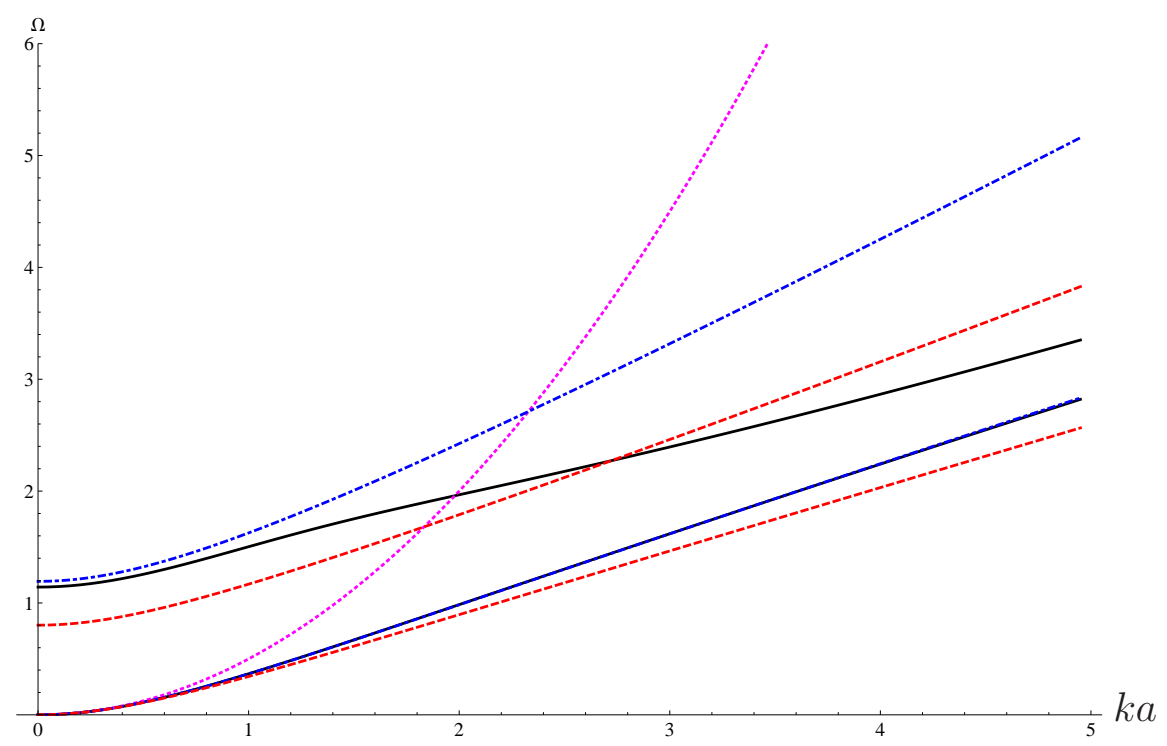

Fig. 3. Dispersion curves for $m=1$ : — Exact, $\cdots$ Euler-Bernoulli, - - - Timoshenko, --- $a^{2}$.

circumferential directions) while $n-2$ are BCs in the normal direction.

For a cylinder simply supported at both ends, mixed boundary conditions are stated as $\hat{u}=\hat{v}=0$ and $\hat{t}_{z z}=0$ at $z=0, L$. This implies that the corresponding series expansion terms are all zero. The three lowest eigenfrequencies for $L / a=10$ and $L / a=2$ are presented for the bending dominant mode in Table 2 and the shear dominant mode in Table 3. It is clear from both tables that the series expansion results converge to the exact results as the power series orders are increased. It is clear from Table 2 that more accurate results are obtained for lower frequencies and slender cylinders. In the light of this statement, it is quite surprising to see the high accuracy for the thick cylinder when compared to the slender cylinder in Table 3. The Timoshenko theory is astonishingly accurate for the bending mode Table 2, but considerably less so for the shearing mode Table 3 . As noted above in Section 9.1, this behavior relates to the choice of shear correction factor. The results for the Euler-Bernoulli theory confirm the well known fact that this theory renders reasonably accurate results for slender beams in the low frequency spectra.

Consider next the cases of clamped-clamped and free-free cylinders. Naturally, these end boundary conditions correspond to zero series expansion displacement terms and zero series expansion stress terms, respectively. The three lowest eigenfrequencies for $L / a=4$ are presented for the bending dominant mode in Table 4. Here the series expansion theory also includes $n=7$ due to the slower convergence in the clamped case. These results show that the Euler-Bernoulli theory does not estimate these frequencies accurately due to the low $L / a$ value. As for the Timoshenko theory, the clamped-clamped case renders very accurate results, while the discrepancy is more pronounced for the free-free case. The opposite situation holds for the series expansion results, where the free-free case is superior to the clamped-clamped case. The exact frequencies are taken from Liew and Hung [13] 


\begin{tabular}{ccccccccc}
$L / a$ & $\Omega$ & Exact & EB & \multicolumn{1}{c}{$\mathrm{T}$} & \multicolumn{1}{c}{$n=3$} & \multicolumn{1}{c}{$n=4$} & \multicolumn{1}{c}{$n=5$} & \multicolumn{1}{c}{$n=6$} \\
\hline \hline & $\Omega_{1}$ & 0.047215 & 0.049348 & 0.047214 & 0.046910 & 0.047214 & 0.047215 & 0.047215 \\
\multirow{2}{*}{10} & $\Omega_{2}$ & 0.16955 & 0.19739 & 0.16952 & 0.16619 & 0.16950 & 0.16955 & 0.16955 \\
& $\Omega_{3}$ & 0.33422 & 0.44413 & 0.33404 & 0.32308 & 0.33388 & 0.33422 & 0.33422 \\
\hline \multirow{3}{*}{2} & $\Omega_{1}$ & 0.71391 & 1.2337 & 0.71310 & 0.67335 & 0.71115 & 0.71381 & 0.71391 \\
& $\Omega_{2}$ & 1.7079 & 4.9348 & 1.7074 & 1.5053 & 1.6748 & 1.7040 & 1.7076 \\
& $\Omega_{3}$ & 2.6765 & 11.103 & 2.6864 & 2.1134 & 2.5227 & 2.6465 & 2.6717
\end{tabular}

Table 2

The eigenfrequencies for $L / a=10$ and $L / a=2$ using exact, Euler-Bernoulli (EB), Timoshenko (T) and the series expansion theories of orders $n=3,4,5,6$ for the bending dominant mode in a cylinder simply supported at $z=0, L$.

\begin{tabular}{cccccccc}
$L / a$ & $\Omega$ & Exact & $\mathrm{T}$ & $n=3$ & $n=4$ & $n=5$ & $n=6$ \\
\hline \hline \multirow{3}{*}{10} & $\Omega_{1}$ & 1.1901 & 1.2470 & 1.0606 & 1.2161 & 1.1888 & 1.1901 \\
& $\Omega_{2}$ & 1.3134 & 1.3892 & 1.1803 & 1.3461 & 1.3116 & 1.3135 \\
& $\Omega_{3}$ & 1.4726 & 1.5862 & 1.3300 & 1.5171 & 1.4701 & 1.4728 \\
\hline \multirow{3}{*}{2} & $\Omega_{1}$ & 1.7841 & 2.0640 & 1.6200 & 1.8551 & 1.7801 & 1.7843 \\
& $\Omega_{2}$ & 2.4584 & 3.4481 & 2.3433 & 2.5066 & 2.4559 & 2.4585 \\
& $\Omega_{3}$ & 3.2266 & 4.9311 & 3.1628 & 3.2551 & 3.2260 & 3.2267
\end{tabular}

Table 3

The eigenfrequencies for $L / a=10$ and $L / a=2$ using exact, Timoshenko (T) and the series expansion theories of orders $n=3,4,5,6$ for the shearing dominant mode in a cylinder simply supported at $z=0, L$.

using the Ritz method. These agree in the free-free case with the results developed by Hutchinson [9] and Leissa and So [12]. For the clamped-clamped case Buchanan and Chua [14] obtained results that are slightly higher than in [13].

\subsection{Mode shapes and stress distributions}

In order to illustrate the differences between the lowest truncation of the current beam theory $(n=3)$, classical theories and the exact theory for the case when $m=1$, various plots on mode shapes and stress distributions are compared for the fundamental frequency for the lowest bending mode for a simply supported cylinder when $L / a=10$. Here all displacement and stress fields are transformed to a cylindrical coordinate system for the classical theories. Note that the circumferential position $\theta$ is chosen so that the trigonometric dependencies $\{\cos \theta ; \sin \theta\}$ are set to unity in all the studied cases. Moreover, the eigenmodes are normalized so that the maximum radial displacement $u$ at $r=a$ is equal 


\begin{tabular}{cccccccccc}
$\mathrm{BC}$ & $\Omega$ & Exact & $\mathrm{EB}$ & $\mathrm{T}$ & $n=3$ & $n=4$ & $n=5$ & $n=6$ & $n=7$ \\
\hline \hline & $\Omega_{1}$ & 0.388 & 0.6992 & 0.3866 & 0.3498 & 0.3768 & 0.3819 & 0.3836 & 0.3844 \\
$\mathrm{CC}$ & $\Omega_{2}$ & 0.773 & 1.9273 & 0.7721 & 0.7008 & 0.7518 & 0.7649 & 0.7686 & 0.7701 \\
& $\Omega_{3}$ & 1.237 & 3.7782 & 1.2312 & 1.1129 & 1.2026 & 1.2241 & 1.2310 & 1.2335 \\
\hline \multirow{3}{*}{$\mathrm{FF}$} & $\Omega_{1}$ & 0.480 & 0.6992 & 0.4798 & 0.4700 & 0.4797 & 0.4797 & 0.4797 & 0.4797 \\
& $\Omega_{2}$ & 0.888 & 1.9273 & 0.8938 & 0.8288 & 0.8869 & 0.8875 & 0.8875 & 0.8875 \\
& $\Omega_{3}$ & 1.297 & 3.7782 & 1.3273 & 1.1831 & 1.2982 & 1.2957 & 1.2970 & 1.2970
\end{tabular}

Table 4

The eigenfrequencies for $L / a=4$ using exact, Euler-Bernoulli (EB), Timoshenko (T) and the series expansion theories of orders $n=3,4,5,6,7$ for the bending dominant mode in a cylinder either clamped or free at both ends.

to unity; that is at $z=L / 2$ for the first eigenmode.

Figure 4(a) illustrates the radial displacement $u$ as a function of the radius for $z=3 L / 4$. For the exact and the present beam theories the radial displacement coincides, while both the Euler-Bernoulli and the Timoshenko theories describe a constant displacement field lying on top of each other. Still the differences between theories are quite small, which partly stem from the way the normalization is defined. The circumferential displacement field $v$ behaves in a similar fashion, not shown here. As for the longitudinal displacement $w$, all theories show a linear behavior for varying $r$ at a fixed $z$. Figure 4(b) displays $w$ as a function of $z$ for $r=a$. Here the Timoshenko curve is almost on top of the coinciding exact and present curves, while the maximum value using the Euler-Bernoulli theory differs from these theories by about $10 \%$.

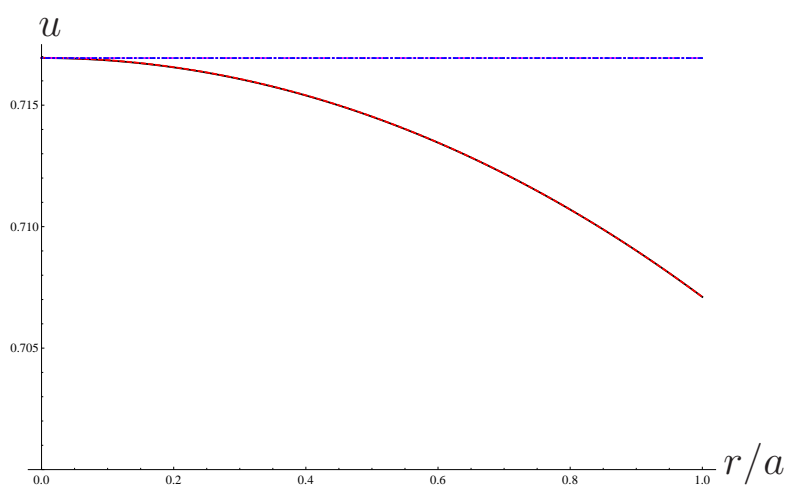

(a) Radial displacement $u$ at $z=3 L / 4$.

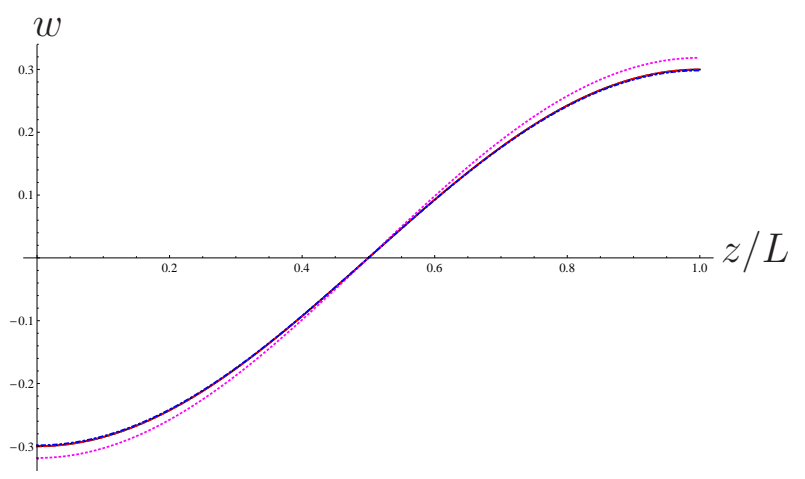

(b) Longitudinal displacement $w$ at $r=a$.

Fig. 4. Displacements for the lowest eigenfrequency. —_ Exact, . . Euler-Bernoulli, - - Timoshenko, -- $n=3$.

Next, consider the stresses as functions of $z$ for $r=a$ in Figure 5. Figure 5(a) for $\sigma_{z z}$ illustrates clearly that both classical theories overestimate the longitudinal normal stress, while the present series curve is virtually on top of the exact curve. Similar results are 
obtained using the classical theories for $\sigma_{\theta \theta}$ and $\sigma_{r r}$, not shown here. In both these latter cases the stresses using the present and exact theories are very small for $\sigma_{\theta \theta}$ and identical to zero for $\sigma_{r r}$, while the maximum stress values at $z=L / 2$ for the classical theories are around half the corresponding longitudinal normal stress values. Note that the lateral boundary conditions constitute the differential equations for the series expansion theories, resulting in $\sigma_{r r} \equiv 0$ at $r=a$ contrary to the classical theories. As for the shear stresses, the magnitudes of these are much smaller than for the normal stresses. The shear stress $\sigma_{\theta z}$ is plotted in Figure 5(b). It is seen that the Timoshenko theory generates slightly smaller stress magnitudes than for the present and exact theories, where the curves almost coincide. The stress magnitudes from the Euler-Bernoulli theory are here zero. The classical theories for $\sigma_{r z}$ behave in similar manner as for $\sigma_{\theta z}$, while the $\sigma_{r \theta}$ stresses are zero for both classical theories (not presented here). Note again that $\sigma_{r \theta}$ and $\sigma_{r z}$ are both identical to zero for the present and the exact theories at the lateral boundary.

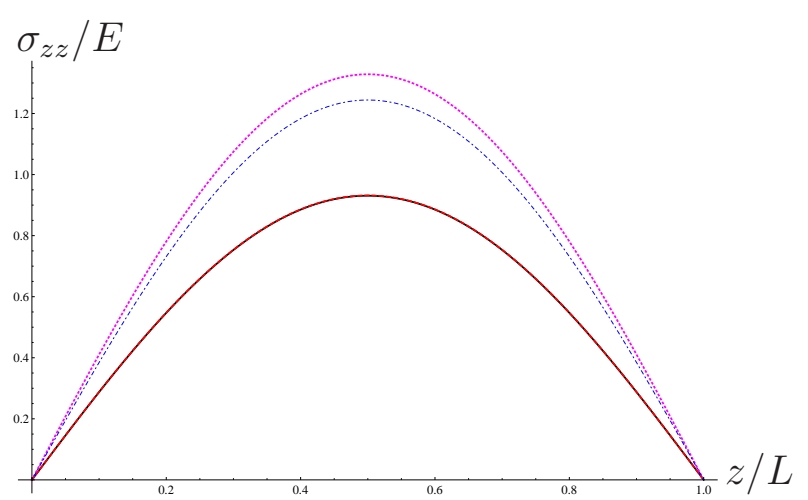

(a) Longitudinal normal stress $\sigma_{z z}$ at $r=a$.

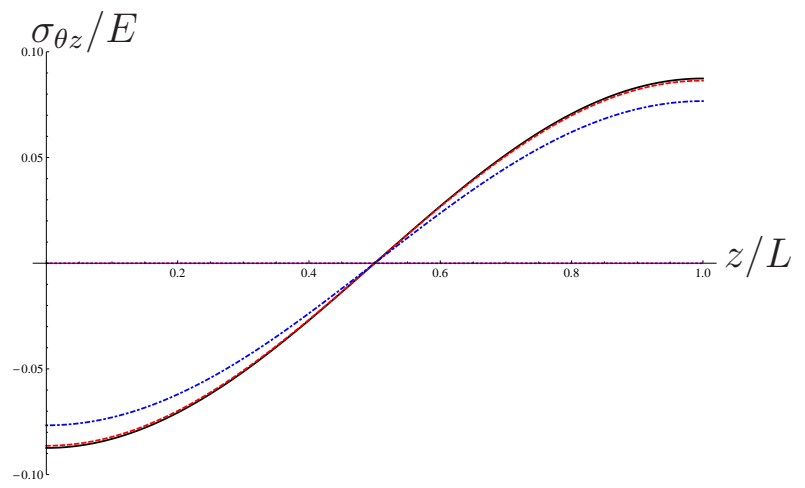

(b) Shear stress $\sigma_{\theta z}$ at $r=a$.

Fig. 5. Stresses for the lowest eigenfrequency. Exact, . . Euler-Bernoulli,

Figure 6 shows the stresses as functions of $r$ for $z=3 L / 4$. The normal stress $\sigma_{z z}$ in Figure 6(a) illustrates that the classical theories overestimate this stress, using the present normalization. As before, the exact and present curves are on top of each other. Similar results are obtained using the classical theories for $\sigma_{\theta \theta}$ and $\sigma_{r r}$, while these stresses using the present and exact theories are very small for all radii. The shear stress $\sigma_{r z}$ is plotted in Figure 6(b). It is seen that the Timoshenko theory generates a constant shear stress, while the stress magnitudes from the Euler-Bernoulli theory are zero. Note that the shear stress using the present theory is zero at the lateral boundary, as expected. The present and exact theories for $\sigma_{\theta z}$ and $\sigma_{r \theta}$ render stress levels that are similar to $\sigma_{r z}$. Both classical theories show similar behavior for $\sigma_{\theta z}$ as for the $\sigma_{r z}$ case, while the $\sigma_{r \theta}$ stress is zero in both theories. 


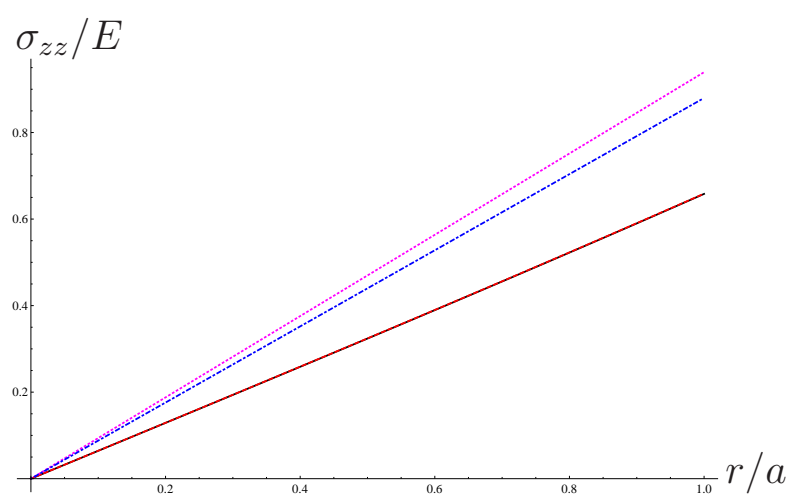

(a) Longitudinal normal stress $\sigma_{z z}$ at $z=3 L / 4$.

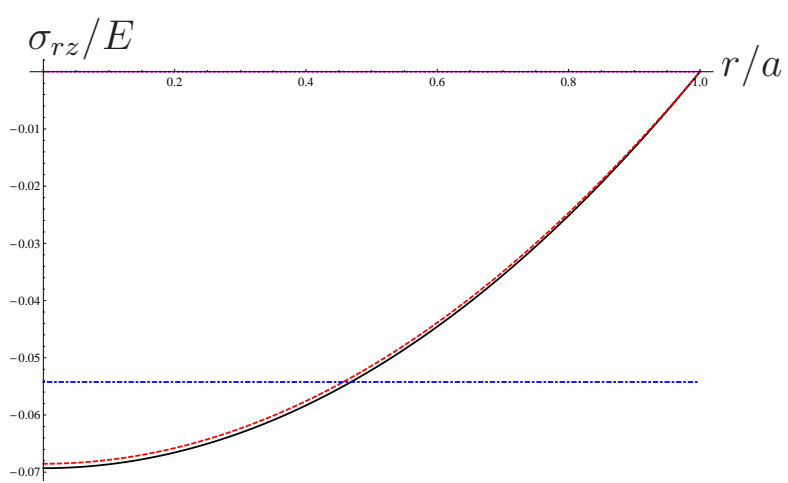

(b) Shear stress $\sigma_{r z}$ at $z=3 L / 4$.

Fig. 6. Stresses for the lowest eigenfrequency. —_Exact, … Euler-Bernoulli, -.- Timoshenko, - - $n=3$.

\subsection{Boundary value problems}

In order to study the effects from non-homogeneous boundary conditions, consider fix frequency problems for prescribed lateral and end boundary conditions, respectively.

For prescribed lateral boundary conditions, assume a transverse beam $(m=1)$ for given tractions $\hat{t}_{i, 1}(z, t)$ in line with $(38)-(40)$. Consider the case of a vertical distributed force acting on a simply supported cylinder, where $-\hat{t}_{r, 1}(z, t)=\hat{t}_{\theta, 1}(z, t)=Q \sin (\pi z / L) \cos (\omega t)$ and $\hat{t}_{z, 1}(z, t)=0$ using the notation in (34). This loading results in a purely vertical traction $Q \sin (\pi z / L) \cos (\omega t)$, resulting in the vertical force per cylinder length $2 \pi a Q \sin (\pi z / L) \cos (\omega t)$. The vertical displacement for the line $r=0$ may thus be expressed $d(z, t)=d \sin (\pi z / L) \cos (\omega t)$ for each theory. Solving the coupled equation system (38)-(40) for various truncation orders, these results may be compared to the exact three dimensional theory and classical engineering theories. Table 5 shows the quotient $d / d_{E B}$ (EB for Euler-Bernoulli) when $L / a=10$ and $L / a=4$ in the static case $(\omega=0)$ using Timoshenko, series expansion and exact theories. It is seen that the Timoshenko theory is considerably more accurate than the Euler-Bernoulli theory, especially for the shorter cylinder. Considering series expansion theory, the results are seen to converge to the exact values. Accurate results are obtained for $n=4$ and higher. Similar solutions may also be obtained using the theory presented in [26], resulting in displacements that are inferior to the Timoshenko theory (not presented here). Note in this static case that the exact results may not be obtained directly from the static counterpart of the dynamic solution presented in Section 7.2. Instead, the methods presented in $[43,44]$ are to be used.

In the case of prescribed end boundary conditions, assume a torsional bar $(m=0)$ with given displacements $\hat{v}_{0,0}(r, t)$ at $z=0$ and $\hat{v}_{0, L}(r, t)$ at $z=L$. Consider the case where the left end is fixed, $\hat{v}_{0,0}(r, t)=0$, and the right end condition is $\hat{v}_{0, L}(r, t)=v_{L} \sin (p r) \cos (\omega t)$. The torsional equation of motion for a laterally free cylinder is given explicitly in (45) for $\hat{t}_{\theta, 0}=0$. Only odd terms are to be used: $v_{2 k+3,0}$ for the equation of motion and $v_{2 k+1,0}$ 


\begin{tabular}{ccccccc}
$L / a$ & Exact & $\mathrm{T}$ & $n=3$ & $n=4$ & $n=5$ & $n=6$ \\
\hline 10 & 1.0685 & 1.0693 & 1.0832 & 1.0686 & 1.0685 & 1.0685 \\
4 & 1.4035 & 1.4334 & 1.4985 & 1.4060 & 1.4035 & 1.4035
\end{tabular}

Table 5

The vertical displacement $d / d_{E B}$ at $r=0$ for $L / a=10$ and $L / a=4$ using exact, Timoshenko (T) and the series expansion theories of orders $n=3,4,5,6$ in a cylinder simply supported at $z=0, L$.

for the end boundary conditions as outlined in Section 6. By expanding the $\hat{v}_{0, L}(r, t)$ in Zernike polynomials $R_{2 k+1}^{1}(r / a)$ in line with (44), the end boundary condition for different truncation orders are presented in Figure $7(\mathrm{a})$. The linear term $k=0$ corresponds to the result for elementary theory. Clearly $\hat{v}_{0, L}(r, t)$ may in this case be modeled accurately using a few terms. By solving (49) adopting the expanded end boundary conditions, the radially series expanded fields $v(r, z) \cos (\omega t)$ are obtained for different truncation orders. Figure 7(b) presents the displacement at $r=a / 2$ for $L / a=10$ and $\omega=c_{E} / a$. Here the exact results are obtained by a Fourier expansion in Bessel function $J_{1}$. By increasing the power series order $k$ in the present theory, the behavior close to $z=L$ is captured more accurately. The result using $k=4$ is virtually indistinguishable from the exact curve, and thus not presented here. Note the decaying Saint-Venant edge effect, where the result using the $k=0$ solution render accurate results away from $z=L$.

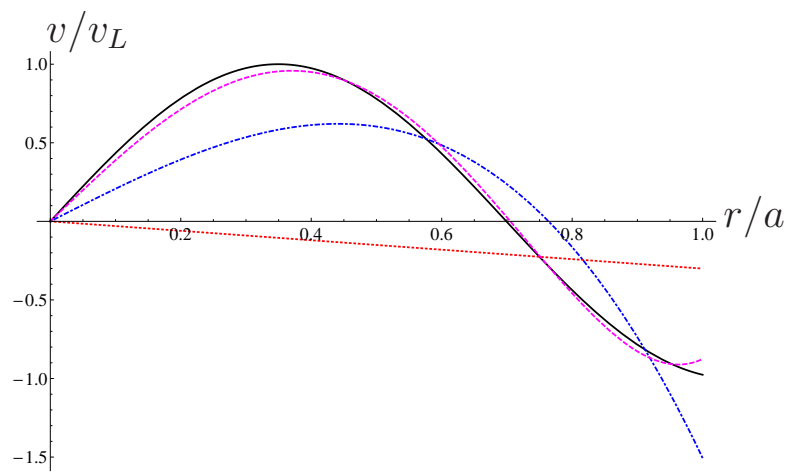

(a) Prescribed displacement $v$ at $z=L$.

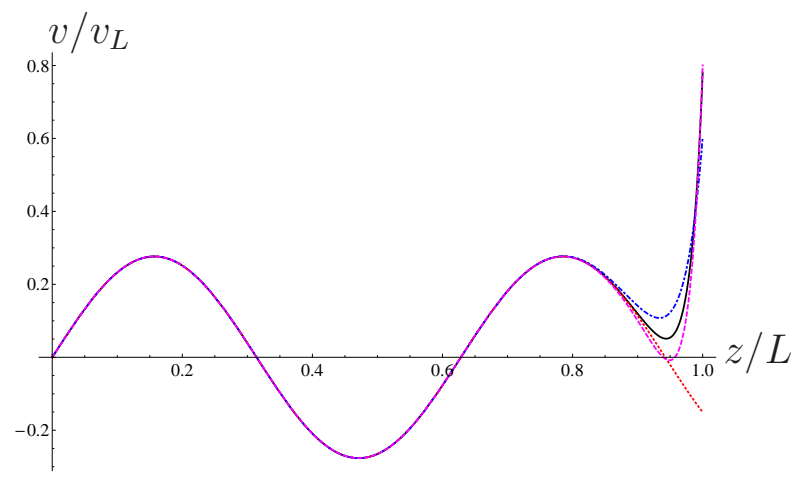

(b) Displacement $v$ at $r=a / 2$.

Fig. 7. Circumferential displacement for different truncation orders. - Exact, $\cdots k=0, \cdots$ $k=1,--k=2$.

\section{Conclusions}

This work presents time domain approximate cylinder equations and corresponding boundary conditions to arbitrary order for different mode families. The method used is based on a power series expansion approach, which together with a generalized Hamilton's principle result in variationally consistent equations that are asymptotically correct to all studied 
orders. Numerical results are presented for various truncation orders and compared to exact and different classical beam theories. For the examples studied herein, a modest truncation order is mostly sufficient to render results that are of high accuracy. Although the present work does not aim at presenting explicit benchmark solutions, it is likely that the systematically developed cylinder equations are capable of rendering accurate solutions for more involved cylinder problems. As for the present lower order truncation theory and the traditional beam theories, these are all fairly adequate for calculating the lower eigenfrequencies, while the results deteriorate for higher eigenfrequencies, less slender cylinders and higher circumferential mode numbers. The Timoshenko theory is very accurate for the bending frequency analyzes, for which the present theory calls for additional series expansion terms for equal accuracy. However, the present lowest order theories are superior to the Timoshenko theory when it comes to mode shapes, the stress distributions and results for higher mode families such as the shearing dominant mode. The differences are most prominent for the stress distributions where it is somewhat surprising that the present lowest order theory gives curves that in most cases are virtually indistinguishable from the exact results.

One application of the new set of cylinder theories is to implement it in finite element codes. Hereby one benefits from the accurate results using one of the present lower order theories, and at the same time the number of elements can be heavily reduced compared to using three dimensional elements. Another issue is to develop higher order theories for more complicated material configurations, such as for functionally graded, porous or micro materials. For such configurations, several different beam theories of both Euler-Bernoulli and Timoshenko type have appeared, and the present systematic approach would render equations that are variationally consistent and directly based on the three dimensional theory of elastodynamics. Hereby equations of various truncation order may be derived that render more accurate results than standard engineering theories. By increasing the truncations to high order, three dimensional benchmark results may then be obtained [36]. Related work based on power series expansion and recursion relations have previously been carried out on porous [45] and functionally graded [36] plates, and is currently directed towards piezoelectric plates, micro plates and functionally graded cylinders.

\section{References}

[1] L. Pochhammer, Über die fortpflanzungsgeschwindigkeiten kleiner schwingungen in einem unbegrenzten isotropen kreiszylinder, Z. Reine Angew. Math. 81 (1876) 324336.

[2] D. Chree, The equations of an isotropic elastic cylinder in polar and cylindrical coordinates, their solutions and applications, Trans. Camb. Phil. Soc. 14 (1889) 250.

[3] G.E. Hudson, Dispersion of elastic waves in solid circular cylinders, Phys. Rev. 63 (1943) 46-51. 
[4] H.N. Abramson, Flexural waves in elastic beams of circular cross section, J. Acoust. Soc. Am. 29 (1957) 42-46.

[5] Y.-H. Pao, R.D. Mindlin, Dispersion of flexural waves in an elastic, circular cylinder, J. Appl. Mech. 27 (1960) 513-520.

[6] G.P. DeVault, C.W. Curtis, Elastic cylinder with free lateral surface and mixed timedependent end conditions, J. Acoust. Soc. Am. 34 (1962) 421-432.

[7] J. McKenna, P.G. Simpkins, Elastic cylinder with free lateral surface and mixed time-dependent end conditions, J. Acoust. Soc. Am. 78 (1985) 1675-1683.

[8] J.R. Hutchinson, Vibrations of solid cylinders, J. Appl. Mech. 47 (1980) 901-907.

[9] J.R. Hutchinson, Transverse vibrations of beams, exact versus approximate solutions, J. Appl. Mech. 48 (1981) 923-928.

[10] A. Bayón, F. Gascón, R. Medina, F.J. Nieves, F. Salazar, Study of pure transverse motion in free cylinders and plates in flexural vibration by Ritz's method, Eur. J. Mech., A/Solids 30 (2011) 423-431.

[11] A.W. Leissa, J. So, Comparisons of vibration frequencies for rods and beams from one-dimensional and three-dimensional analyses, J. Acoust. Soc. Am. 98 (1995) 21222135.

[12] A.W. Leissa, J. So, Accurate vibration frequencies of circular cylinders from threedimensional analysis, J. Acoust. Soc. Am. 98 (1995) 2136-2141.

[13] K.M. Liew, K.C. Hung, Three-dimensional vibratory characteristics of solid cylinders and some remarks on simplified beam theories, Int. J. Solids Struct. 32 (1995) 34993513.

[14] G.R. Buchanan, C.-L. Chua, Frequencies and mode shapes for finite length cylinders, J. Sound Vib. 246 (2001) 927-941.

[15] S.P. Timoshenko, On the correction for shear of the differential equation for transverse vibrations of prismatic bars, Phil. Mag. XLI (1921) 744-746. Reprinted in The Collected Papers of Stephen P. Timoshenko, McGraw-Hill, London 1953.

[16] W.B. Bickford, A consistent higher order beam theory, Developments in Theoretical and Applied Mechanics 1 (1982) 137-150.

[17] P.R. Heyliger, J.N. Reddy, A higher order beam finite element for bending and vibration problems, J. Sound Vib. 126 (1988) 309-326.

[18] T. Kant, A. Gupta, Analysis of orthotropic plates based on three theories by segmentation method, J. Sound Vib. 125 (1988) 193-202.

[19] M. Levinson, A new rectangular beam theory, J. Sound Vib. 74 (1981) 81-87.

[20] H. Matsunaga, Free vibration and stability of thin elastic beams subjected to axial forces, J. Sound Vib. 191 (1996) 917-933.

[21] J.N. Reddy, C.M. Wang, K.H. Lee, Relationships between bending solutions of classical and shear deformation beam theories, Int. J. Solids Struct. 34 (1997) 3373-3384.

[22] G. Shi, G.Z. Voyiadjis, A sixth-order theory of shear deformable beams with variational consistent boundary conditions, J. Appl. Mech. 78 (2011) 021019 1-11.

[23] K.P. Soldatos, C. Sophocleous, On shear deformable beam theories The frequency and normal mode equations of the homogeneous othotropic Bickford beam, J. Sound Vib. 242 (2001) 215-245. 
[24] J.D. Achenbach, S.J. Fang, Asymptotic analysis of the modes of wave propagation in a solid cylinder, J. Acoust. Soc. Am. 47 (1970) 1282-1289.

[25] G. Dökmezi, A general theory of elastic beams, Int. J. Solids Structures 8 (1970) 1205-1222.

[26] H. Fan, G.E.O. Widera, Refined engineering beam theory based on the asymptotic expansion approach, AIAA J. 29 (1991) 444-449.

[27] J.B. Kosmatka, Transverse vibrations of shear-deformable beams using a general higher order theory, J. Sound Vib. 160 (1993) 259-277.

[28] P.A. Martin, On flexural waves in cylindrically anisotropic elastic rods, Int. J. Solids Struct. 42 (2005) 2161-2179.

[29] I.K. Silverman, Flexural vibrations of circular beams, J. Sound Vib. 210 (1998) 661-672.

[30] P.D. Folkow, K. Mauritsson, Dynamic higher-order equations for finite rods, Q. J1 Mech. Appl. Math. 63 (2010) 1-22.

[31] A. Boström, On wave equations for elastic rods, Z. Angew. Math. Mech. 80 (2000) $245-251$.

[32] A. Boström, G. Johansson, P. Olsson, On the rational derivation of a hierarchy of dynamic equations for a homogeneous, isotropic, elastic plate, Int. J. Solids Struct. 38 (2001) 2487-2501.

[33] A.M. Hägglund, P.D. Folkow, Dynamic cylindrical shell equations by power series expansions, Int. J. Solids Struct. 45 (2008) 4509-4522.

[34] N.A. Losin, Asymptotics of flexural waves in isotropic elastic plates, J. Appl. Mech. 64 (1997) 336-342.

[35] K. Mauritsson, P.D. Folkow, A. Boström, Dynamic equations for a fully anisotropic elastic plate, J. Sound Vib. 330 (2011) 2640-2654.

[36] S.S. Vel, R.C. Batra, Three-dimensional exact solution for the vibration of functionally graded rectangular plates, J. Sound Vib. 272 (2004) 703-730.

[37] R.B. Hetnarski, J. Ignaczak, Mathematical theory of elasticity Taylor \& Francis, 2004.

[38] K. Washizu, Variational methods in elasticity and plasticity Pergamon Press, 1982.

[39] J.D. Achenbach, Wave Propagation in Elastic Solids North-Holland, Amsterdam, 1973.

[40] M. Born, E. Wolf, Principles of optics Cambridge University Press, Cambridge, 1999.

[41] N.A. Losin, On the equivalence of dispersion relations resulting from Rayleigh-Lamb frequency equation and the operator plate model, J. Vibr. Acoust. 123 (2001) 417420.

[42] T. Kaneko, On Timoshenko's correctionfor shear in vibrating beams, J. Appl. Phys. 8 (1975) 1927-1936.

[43] Y.P. Chiu, A.S. Weinstein, C.F. Zorowski, The stresses, deformations and bending of a simply supported cylinder subjected to a symmetric normal surface loading, Int. J. Mech. Sci. 5 (1963) 119-136.

[44] D. Palaniappan, A general solution of equations of equilibrium in linear elasticity, Appl. Math. Model. 35 (2011) 5494-5499. 
[45] P.D. Folkow, M. Johansson, Dynamic equations for fluid-loaded porous plates using approximate boundary conditions, J. Acoust. Soc. Am. 125 (2009) 2954-2966. 